\title{
Resolvent dynamical systems and mixed variational inequalities
}

\author{
Bandar Bin-Mohsin ${ }^{a}$, Muhammad Aslam Noor ${ }^{a, b, *}$, Khalida Inayat Noor ${ }^{b}$, Rafia Latif ${ }^{b}$ \\ ${ }^{a}$ Department of Mathematics, King Saud University, Riyadh, Saudi Arabia. \\ ${ }^{b}$ Department of Mathematics, COMSATS Institute of Information Technology, Islamabad, Pakistan.
}

Communicated by A. Atangana

\begin{abstract}
In this paper, we use the dynamical systems technique to suggest and investigate some inertial proximal methods for solving mixed variational inequalities and related optimization problems. It is proved that the convergence analysis of the proposed methods requires only the monotonicity. Some special cases are also considered. Our method of proof is very simple as compared with other techniques. Ideas and techniques of this paper may be extended for other classes of variational inequalities and equilibrium problems. (C) 2017 All rights reserved.
\end{abstract}

Keywords: Variational inequalities, dynamical systems, inertial proximal methods, convergence.

2010 MSC: 26D15, 26D10, 90C23, 49J40.

\section{Introduction and Preliminaries}

Variational inequalities, which were introduced and investigated by Stampacchia [30]. Variational inequalities can be viewed as significant and natural generalization of the variational principles, the origin of which can be traced back to Euler, Lagrange, Newton and Bernoulli's brothers. It is remarkable and amazing that a wide class of unrelated problems, which in pure, applied and engineering sciences can be studied in the general and unified framework of variational inequalities. The ideas and techniques of this theory are being applied in a variety of diverse areas of pure and applied sciences and proved to innovative, see [1-4, 6-11, 13, 15-17, 19, 20, 22, 24, 26-33].

Variational inequalities involving the nonlinear term is called the mixed variational inequality or variational inequality of the second kind. Mixed variational inequalities have applications in elasticity, structural engineering and electronic network, see [12] and the references therein. Due to the presence of the nonlinear term, the projection method and its variant form can be used to establish the equivalence between the mixed variational inequalities and the fixed point problem. However, if the nonlinear term is a proper, convex and lower-semi continuous, then one can show that the mixed variational inequalities are equivalent to the fixed point problem using the resolvent operator technique. This alternative formulation

\footnotetext{
*Corresponding author

Email addresses: balmohsen@ksu.edu.s (Bandar Bin-Mohsin), noormaslam@gmail.com (Muhammad Aslam Noor), khalidanoor@hotmail . com (Khalida Inayat Noor), rafialatif818@gmail .com (Rafia Latif)
}

doi:10.22436/jnsa.010.06.07 
has played an important part in studying the existence of solution and developing several numerical iterative methods for solving variational inequalities and complementarity problems. One can again use the fixed point formulation to suggest the explicit and implicit method for solving the variational inequalities. To implement the implicit type method, one usually use the predictor-corrector technique. Consequently, the implicit methods is equivalent to the extraresolvent method. The extraresolvent method can be viewed as analogous to the extragradient method, which was suggested and investigated by Korpelevich [14]. It is known that if the solution exists, then the convergence of the implicit method needs only that the operator is monotone and Lipschitz continuous. In some cases, it is very difficult to find the Lipschitz continuity constant. Noor [24] proved that the convergence of the extragradient method of Korpelevich [14] only needs psuedomonotonicity, using the auxiliary principle technique. This can be viewed as a significant and important refinement of the previously known results. Using the fixed point formulation, Noor [24] and Noor et al. [25] suggested an inertial proximal method for solving mixed variational inequalities. Such type of the inertial proximal methods were suggested by Alvarez and Attouch [1] for solving the variational inclusions involving the sum of the operator. It has been shown by Noor [24] that the convergence of inertial proximal methods can be proved under the monotonicity of the operator.

Dynamical systems arise naturally in numerous applied and theoretical fields including celestial mechanics, financial forecasting, environmental applications, neuroscience and brain modeling. The fixed point formulation of the variational inequalities has been used to suggest two type of dynamical systems. The first one, is due to Friesz et al. [9] is designated as global projected dynamical systems, whereas the second which is mainly due to Dupuis and Nagurney [8] is called the local dynamical system. We would like to mention that novel and innovative features of these projected dynamical systems is that the set of the stationary points of the dynamical systems correspond to the set of the solutions of the variational inequalities. This shows that all the problems which can be studied in the general framework of variational inequalities can be studied in the framework of the dynamical systems. Consequently, various numerical methods which have been developed for solving dynamical systems can be used to find the approximate solution of the variational inequalities. For example, neural network techniques have been used for solving the variational inequalities, see Xia and Wang [32]. For more details on the applications of the dynamical systems, see [7-9, 11, 15, 16, 18, 20, 22, 24, 26-33].

In this paper, we use the dynamical systems approach for the construction of the inertial proximal methods for solving the mixed variational inequalities. We use the fixed point formulation to suggest a second-order resolvent dynamical system associated with the mixed variational inequalities. We use the variant form of finite difference schemes to discretize the second derivative to suggest some inertial proximal methods for solving the variational inequalities. We use the resolvent lemma to express the inertial proximal methods as an auxiliary mixed variational inequality. This reformulation is used to analyze the convergence criteria of the proposed inertial proximal method under the monotonicity of the operator. In our analysis, we do not need any resolvent operator, which is a novel way to prove the convergence of the inertial proximal methods for solving variational inequalities.

\section{Preliminaries and basic results}

Let $\mathrm{H}$ be a real Hilbert space, whose norm and inner product are denoted by $\|\cdot\|$ and $\langle\cdot, \cdot\rangle$ respectively. For a given operator $\mathrm{G}: \mathrm{H} \rightarrow \mathrm{H}$ and a proper, convex and lower-semi continuous function $\mathrm{f}: \mathrm{H} \longrightarrow$ $\mathbb{R} \cup\{\infty\}$, consider a problem of finding $u \in H$, such that

$$
\langle\mathrm{G}(\mathrm{u}), v-\mathrm{u}\rangle+\mathrm{f}(v)-\mathrm{f}(\mathrm{u}) \geqslant 0, \quad \forall v \in \mathrm{H},
$$

which is called mixed variational inequality problem. For the applications, formulation and other aspects of the mixed variational inequalities, see $[4,6-11,13,15,16,18,20,22,24-33]$ and the references therein. We note that if $f$ is the indicator function of a closed convex set $\mathrm{K}$ in $\mathrm{H}$, then problem (2.1) reduces to finding $u \in K$, such that

$$
\langle\mathrm{G}(\mathrm{u}), v-\mathrm{u}\rangle \geqslant 0, \quad \forall v \in \mathrm{K},
$$


which is known as the variational inequality, introduced and studied by Stampacchia [30]. For the recent applications, formulation numerical methods and other aspects of variational inequalities, see [4, 6-11, $13,15,16,18,20,22,24-33]$.

Now we define the following concepts.

Definition 2.1. The nonlinear operator $\mathrm{G}: \mathrm{H} \rightarrow \mathrm{H}$ is called

(i) Strongly monotone, if there exists a constant $\alpha>0$, such that

$$
\langle G(u)-G(v), u-v\rangle \geqslant \alpha\|u-v\|^{2}, \quad \forall u, v \in H .
$$

(ii) Monotone, if

$$
\langle\mathrm{G}(\mathrm{u})-\mathrm{G}(v), \mathrm{u}-v\rangle \geqslant 0, \quad \forall \mathrm{u}, v \in \mathrm{H} .
$$

Remark 2.2. It is known that strongly monotonicity implies monotonicity but the converse is not true. This means that monotonicity is a weaker condition than strongly monotonicity.

Definition 2.3. If $G$ is a maximal monotone operator on $H$, then for a constant $\rho>0$, the resolvent operator denoted by $\mathrm{J}_{\mathrm{G}}($.$) is defined as$

$$
\mathrm{J}_{\mathrm{G}}(\mathrm{u})=(\mathrm{I}+\rho \mathrm{G})^{-1}(\mathrm{u}), \quad \forall \mathrm{u} \in \mathrm{H},
$$

where I is the identity operator.

It is known that a monotone operator $\mathrm{G}$ is maximal, if and only if its resolvent operator $\mathrm{J}_{\mathrm{G}}$ is defined everywhere. Also, the resolvent operator $\mathrm{J}_{\mathrm{G}}$ is nonexpansive, that is

$$
\left\|\mathrm{J}_{\mathrm{G}}(\mathrm{u})-\mathrm{J}_{\mathrm{G}}(v)\right\| \leqslant\|\mathrm{u}-v\|, \quad \forall \mathrm{u}, v \in \mathrm{H} .
$$

Remark 2.4. It is known that the subdifferential $\partial \mathrm{f}$ of a proper, convex and lower semi-continuous function $f$ is a maximal monotone operator. Thus, we define by

$$
\mathrm{J}_{\mathrm{f}}(\mathrm{u})=(\mathrm{I}+\rho \partial \mathrm{f})^{-1}(\mathrm{u}), \quad \forall \mathrm{u} \in \mathrm{H},
$$

the resolvent operator associated with $\partial f$ and $\rho>0$ is a constant.

We also need the well-known result, which is known as the resolvent lemma. This result plays crucial role in the convergence analysis of the proposed inertial proximal methods.

Lemma 2.5. Let $\mathrm{f}$ be a proper, convex and lower semicontinuous function. For a given $z \in \mathrm{H}, \mathrm{u} \in \mathrm{K}$ satisfies

$$
\langle u-z, u-v\rangle+\rho f(v)-\rho f(u) \geqslant 0, \quad \forall v \in H,
$$

if and only if

$$
\mathrm{u}=\mathrm{J}_{\mathrm{f}}(z),
$$

where $\mathrm{J}_{\mathrm{G}}$ is the resolvent operator associated with the proper, convex and lower semi-continuous function and $\rho>0$ is a constant.

Using Lemma 2.5 one can show that the mixed variational inequality (2.1) is equivalent to the fixed point problem.

Theorem 2.6 ([13]). The element $\mathrm{u} \in \mathrm{K}$ satisfies

$$
\langle\mathrm{G}(\mathrm{u}), v-\mathrm{u}\rangle+\mathrm{f}(v)-\mathrm{f}(\mathrm{u}) \geqslant 0, \quad \forall v \in \mathrm{H},
$$

if and only if

$$
\mathrm{u}=\mathrm{J}_{\mathrm{f}}[\mathrm{u}-\rho \mathrm{G}(\mathrm{u})],
$$

where $\mathrm{J}_{\mathrm{f}}$ is the resolvent operator and $\rho>0$ is a constant. 
From Theorem 2.6, we see that mixed variational inequalities (2.1) are equivalent to the fixed point problem (2.2). This alternate formulation is used to study the existence of a solution of the variational inequalities and to suggest several explicit and implicit methods.

Theorem 2.6 was used to introduce the resolvent dynamical system associated with the mixed variational inequalities (2.1). In fact, we consider the problem of finding $u \in H$ such that

$$
\frac{d u}{d t}=\lambda\left\{J_{f}[u-\rho G(u)]-u\right\}, \quad u\left(t_{0}\right)=u_{o} \in H .
$$

Such type of the dynamical systems were introduced and investigated by Noor [21]. Form the definition, it is clear that solution of the resolvent dynamical system always exists. This implies that qualitative results such as the existence, uniqueness and continuous dependence of the solution of (2.3) can be studied. For more details, see Noor [21, 23].

\section{Main results}

In this section, we use the fixed point formulation to suggest and consider a new second order resolvent dynamical system associated with mixed variational inequalities (2.1). We use this dynamical system to suggest and investigate some inertial proximal methods for solving the variational inequalities (2.1). These inertial implicit methods are constructed using the central finite difference schemes and its variant forms.

To be more precise, we consider the problem of finding $u \in H$ such that

$$
\gamma \ddot{u}+\dot{u}+u=J_{f}[u-\rho G(u)], \quad u\left(t_{0}\right)=\alpha, \dot{u}\left(t_{0}\right)=\beta,
$$

where $\gamma>0$ and $\rho>0$ are constants. Problem (3.1) is called second order dynamical system. If $\gamma=0$, then dynamical system (3.1) is exactly the dynamical system (2.3), which was studied extensively.

We discretize the second-order dynamical systems (3.1) using central finite difference and backward difference schemes to have

$$
\gamma \frac{u_{n+1}-2 u_{n}+u_{n-1}}{h^{2}}+\frac{u_{n}-u_{n-1}}{h}+u_{n+1}=J_{f}\left[u_{n}-\rho G\left(u_{n+1}\right)\right],
$$

where $h$ is the step size.

Using this discrete form, we suggest the following new iterative method for solving the variational inequalities (2.1).

Algorithm 3.1. For a given $\mathrm{u}_{0} \in \mathrm{H}$, compute $\mathrm{u}_{\mathrm{n}+1}$ by the iterative scheme

$$
u_{n+1}=J_{f}\left[u_{n}-\rho G\left(u_{n+1}\right)-\frac{(\gamma) u_{n+1}-(2 \gamma-h) u_{n}+(\gamma-h) u_{n-1}}{h^{2}}\right], \quad n=0,1,2, \ldots .
$$

Algorithm 3.1 is called the inertial proximal method for solving the mixed variational inequalities and related optimization problems. This is a new proposed method.

We note that for $\gamma=0$, Algorithm 3.1 reduces to the following iterative method for solving the mixed variational inequalities (2.1).

Algorithm 3.2. For a given $\mathrm{u}_{0} \in \mathrm{H}$, compute $\mathrm{u}_{\mathrm{n}+1}$ by the iterative scheme

$$
u_{n+1}=J_{f}\left[u_{n}-\rho G\left(u_{n+1}\right)-\frac{u_{n}-u_{n-1}}{h}\right], \quad n=0,1,2, \cdots .
$$

Using Lemma 2.5, Algorithm 3.1 can be rewritten in the equivalent form as: 
Algorithm 3.3. For a given $\mathrm{u}_{0} \in \mathrm{H}$, compute $\mathrm{u}_{\mathrm{n}+1}$ by the iterative scheme

$$
\begin{gathered}
\left\langle\rho \mathrm{G}\left(\mathrm{u}_{\mathrm{n}+1}\right)+\frac{\left(\gamma+\mathrm{h}^{2}\right) \mathrm{u}_{\mathrm{n}+1}-\left(2 \gamma-\mathrm{h}+\mathrm{h}^{2}\right) \mathrm{u}_{\mathrm{n}}+(\gamma-\mathrm{h}) \mathrm{u}_{\mathrm{n}-1}}{\mathrm{~h}^{2}}, v-u_{n+1}\right\rangle \\
+\rho f(v)-\rho f\left(\mathrm{u}_{\mathrm{n}+1}\right) \geqslant 0, \quad \forall v \in \mathrm{H} .
\end{gathered}
$$

We again discretize the dynamical system (3.1) using the finite difference schemes to have:

$$
\gamma \frac{u_{n+1}-u_{n-1}}{2 h}+\frac{u_{n}-u_{n-1}}{h}+u_{n+1}=P_{K}\left[u_{n}-\rho G\left(u_{n+1}\right)\right],
$$

where $h$ is a step size. This formulation is used to suggest the following iterative method for solving the mixed variational inequalities (2.1) as:

Algorithm 3.4. For a given $\mathrm{u}_{0} \in \mathrm{H}$, compute $\mathrm{u}_{\mathrm{n}+1}$ by the iterative scheme

$$
u_{n+1}=J_{f}\left[u_{n}-\rho G\left(u_{n+1}\right)-\frac{\gamma u_{n+1}+2 u_{n}-(2+\gamma) u_{n-1}}{2 h}\right], \quad n=0,1,2, \cdots .
$$

This is also called the inertial proximal method for solving the variational inequalities and appears to be a new one.

If $\gamma=0$, then Algorithm 3.4 reduces to Algorithm 3.2 for solving the mixed variational inequalities (2.1).

We again discretize the second-order dynamical systems (3.1) using central difference scheme and forward difference scheme to suggest the following inertial proximal method for solving (2.1).

Algorithm 3.5. For a given $\mathrm{u}_{0} \in \mathrm{H}$, compute $\mathrm{u}_{\mathrm{n}+1}$ by the iterative scheme

$$
u_{n+1}=J_{f}\left[u_{n}-\rho G\left(u_{n+1}\right)-\frac{(\gamma+h) u_{n+1}-(2 \gamma+h) u_{n}+\gamma u_{n-1}}{h^{2}}\right], \quad n=0,1,2, \cdots .
$$

Algorithm 3.5 is quite different from other inertial proximal methods for solving the variational inequalities.

If $\gamma=0$, then Algorithm 3.5 collapses to:

Algorithm 3.6. For a given $\mathrm{u}_{0} \in \mathrm{H}$, compute $\mathrm{u}_{\mathrm{n}+1}$ by the iterative scheme

$$
u_{n+1}=J_{f}\left[u_{n}-\rho G\left(u_{n+1}\right)-\frac{u_{n+1}-u_{n}}{h}\right], \quad n=0,1,2, \cdots .
$$

Algorithm 3.6 is a proximal method for solving the mixed variational inequalities. Such type of proximal methods were suggested by Noor [25] using the fixed point problems.

In brief, by suitable descritization of the second-order dynamical systems (3.1), one can construct a wide class of explicit and implicit method for solving inequalities.

We now consider the convergence criteria of Algorithm 3.1 using the technique of Alvarez and Attouch [1] and Noor et al. [25].

Theorem 3.7. Let $\mathrm{u} \in \mathrm{H}$ be the solution of variational inequality (2.1). Let $\mathrm{u}_{\mathrm{n}+1}$ be the approximate solution obtained from (3.2). If $\mathrm{G}$ is monotone, then

$$
\left(h+h^{2}\right)\left\|u-u_{n+1}\right\|^{2} \leqslant\left(\gamma+h^{2}\right)\left\|u-u_{n}\right\|^{2}-\left(\gamma+h^{2}\right)\left\|u_{n+1}-u_{n}\right\|^{2}+(\gamma-h)\left\|u_{n-1}-u_{n}\right\|^{2} .
$$

Proof. Let $u \in \mathrm{H}$ be the solution of variational inequality (2.1). Then

$$
\langle\rho G(v), v-u\rangle+\rho f(v)-\rho f(u) \geqslant 0, \quad \forall v \in H,
$$

since $\mathrm{G}$ is monotone operator. 
Setting $v=u_{n+1}$ in (3.4), we have

$$
\left\langle\rho G\left(u_{n+1}\right), u_{n+1}-u\right\rangle+\rho f\left(u_{n+1}\right)-\rho f(u) \geqslant 0 .
$$

Taking $v=u$ in(3.2), we have

$$
\left\langle\rho G\left(u_{n+1}\right)+\frac{\left(\gamma+h^{2}\right) u_{n+1}-\left(2 \gamma-h+h^{2}\right) u_{n}+(\gamma-h) u_{n-1}}{h^{2}}, u-u_{n+1}\right\rangle+\rho f(u)-\rho f\left(u_{n+1}\right) \geqslant 0 .
$$

From (3.5) and (3.6), we obtain

$$
\left\langle\left(\gamma+h^{2}\right) u_{n+1}-\left(2 \gamma-h+h^{2}\right) u_{n}+(\gamma-h) u_{n-1}, u-u_{n+1}\right\rangle \geqslant 0 .
$$

Thus

$$
\begin{aligned}
0 \leqslant & \left(\gamma+h^{2}\right)\left\langle u_{n+1}-u_{n}, u-u_{n+1}\right\rangle+(\gamma-h)\left\langle u_{n-1}-u_{n}, u-u_{n+1}\right\rangle \\
\leqslant & \left(\gamma+h^{2}\right)\left\|u-u_{n}\right\|^{2}-\left(\gamma+h^{2}\right)\left\|u_{n+1}-u_{n}\right\|^{2}-\left(\gamma+h^{2}\right)\left\|u-u_{n+1}\right\|^{2} \\
& +(\gamma-h)\left\|u_{n-1}-u_{n}\right\|^{2}+(\gamma-h)\left\|u-u_{n+1}\right\|^{2} \\
= & \left(\gamma+h^{2}\right)\left\|u-u_{n}\right\|^{2}-\left(\gamma+h^{2}\right)\left\|u_{n+1}-u_{n}\right\|^{2}+(\gamma-h)\left\|u_{n-1}-u_{n}\right\|^{2} \\
& -h(1+h)\left\|u-u_{n+1}\right\|^{2},
\end{aligned}
$$

where we have used the following inequalities

$$
2\langle u, v\rangle=\|u+v\|^{2}-\|u\|^{2}-\|v\|^{2},
$$

and

From (3.7), we have

$$
2\langle u, v\rangle \leqslant\|u\|^{2}-\|v\|^{2}
$$

$$
\left(h+h^{2}\right)\left\|u-u_{n+1}\right\|^{2} \leqslant\left(\gamma+h^{2}\right)\left\|u-u_{n}\right\|^{2}-\left(\gamma+h^{2}\right)\left\|u_{n+1}-u_{n}\right\|^{2}+(\gamma-h)\left\|u_{n-1}-u_{n}\right\|^{2},
$$

the required (3.3).

Theorem 3.8. Let $u \in H$ be the solution of variational inequality (2.1). Let $u_{n+1}$ be the approximate solution obtained from (3.2). If $\mathrm{G}$ is monotone operator, then $\mathrm{u}_{\mathrm{n}+1}$ converges to $\mathrm{u} \in \mathrm{H}$ satisfying (2.1).

Proof. Let $u \in H$ be a solution of (2.1). From (3.3), it follows that the sequence $\left\{\left\|u-u_{i}\right\|\right\}$ is non-increasing and consequently, $\left\{u_{n}\right\}$ is bounded. Also from (3.3), we have

$$
\sum_{i=1}^{\infty}\left\|u_{n}-u_{n+1}\right\|^{2} \leqslant\left\|u-u_{1}\right\|^{2}+\frac{\gamma-h}{\gamma+h^{2}}\left\|u_{0}-u_{1}\right\|^{2},
$$

which implies that

$$
\lim _{n \rightarrow \infty}\left\|u_{n+1}-u_{n}\right\|^{2}=0 .
$$

Since sequence $\left\{u_{i}\right\}_{i=1}^{\infty}$ is bounded, so there exists a cluster point $\hat{u}$ to which the subsequence $\left\{u_{i k}\right\}_{k=i}^{\infty}$ converges. Replacing $u_{n}$ by $u_{n_{i}}$ in (3.2) and taking the limit as $n_{j} \longrightarrow \infty$, we have

$$
\langle\mathrm{G}(\hat{\mathrm{u}}), v-\hat{\mathrm{u}}\rangle+\rho \mathrm{f}(\hat{\mathrm{u}})-\rho \mathrm{f}(\mathrm{u}) \geqslant 0, \quad \forall v \in \mathrm{H},
$$

which implies that $\hat{u}$ solves (2.1) and

$$
\left\|u_{n+1}-u\right\|^{2} \leqslant \frac{\gamma+h^{2}}{h+h^{2}}\left\|u-u_{n}\right\|^{2}+\frac{\gamma-h}{h+h^{2}}\left\|u_{n}-u_{n-1}\right\|^{2} \leqslant\left\|u-u_{n}\right\|^{2} .
$$

Using this inequality, one can show that the cluster point $\hat{u}$ is unique and

$$
\lim _{n \rightarrow \infty} u_{n+1}=\hat{u} \text {. }
$$




\section{Extensions and future work}

We would like to mention that the results obtained in this paper may be extended for strongly mixed variational inequalities, which were introduced and studied by Bin-Mohsin et al. [5]. More precisely, we consider the problem of finding $\mathrm{u} \in \mathrm{H}$ such that

$$
\langle\mathrm{G}(\mathrm{u}), v-\mathrm{u}\rangle+\mathrm{f}(v)-\mathrm{f}(\mathrm{u}) \geqslant \mu\|v-\mathrm{u}\|^{2}, \quad \forall v \in \mathrm{H},
$$

which is called the strongly mixed variational inequality. It has been shown that the minimum of the sum of differentiable convex function and non-differentiable strongly convex function can be characterized by the strongly mixed variational inequalities. Since the subdifferential $\partial f($.$) associated with the proper,$ strongly convex and semi-lower semi-continuous function $f$ is strongly maximal monotone, one can define the resolvent operator $J_{s f}$ as:

$$
\mathrm{J}_{s f}(\mathrm{u})=(\mathrm{I}+\rho \mu \partial \mathrm{f})^{-1}(\mathrm{u}), \quad \forall \mathrm{u} \in \mathrm{H} .
$$

Using this resolvent operator, one can establish the equivalence between the strongly mixed variational inequalities and the fixed point problem, that is,

Lemma 4.1. $u \in \mathrm{H}$ is a solution of the strongly mixed variational inequality (4.1), if and only if, $\mathrm{u} \in \mathrm{H}$ satisfies the equation

$$
u=J_{s f}[u-\rho T u]
$$

where $\mathrm{J}_{\mathrm{sf}}()=.(\mathrm{I}+\rho \mu \partial \mathrm{f})^{-1}($.$) is the resolvent operator.$

It has been proved that the resolvent operator $\mathrm{J}_{s f}$ is Lipschitz constant with constant $\frac{1}{1+\rho \mu}$, where $\mu>0$ is the constant of the strongly convex function $f$.

Using this equivalent formulation, one can consider the second-order dynamical systems as:

Find $u \in H$ such as:

$$
\gamma \ddot{\mathfrak{u}}+\dot{\mathfrak{u}}+\mathfrak{u}=J_{s f}[\mathfrak{u}-\rho \mathrm{G}(\mathfrak{u})], \quad \mathfrak{u}\left(\mathrm{t}_{0}\right)=\alpha, \dot{u}\left(t_{0}\right)=\beta,
$$

where $\gamma>0$ and $\rho>0$ are constants.

We would like to point out that all the results obtained in this paper continue to hold for the strongly mixed variational inequalities and dynamical systems. For more details, see Bin-Mohsin et al. [5].

\section{Conclusion}

In this paper, we have used a second-order resolvent dynamical systems to suggest some inertial proximal methods for solving mixed variational inequalities. The convergence analysis of these methods have been considered under some weaker conditions. Our method of convergence is very simple as compared with other techniques. Comparison and implementation of these new methods need further efforts. We have only discussed that the dynamical systems can be used to suggest some iterative for finding the approximate solutions of the variational inequalities. The ideas and techniques presented in this paper may be starting point for further developments. Much work is needed to implement this technique.

\section{Acknowledgment}

The authors would like to thank Rector, COMSATS Institute of Information Technology, Pakistan, for providing excellent research and academic environment. The authors are pleased to acknowledge the support of "the support of Distinguished Scientist Fellowship Program(DSFP), King Saud University, Riyadh, Saudi Arabia". 


\section{References}

[1] F. Alvarez, H. Attouch, An inertial proximal method for maximal monotone operators via discretization of a nonlinear oscillator with damping, Wellposedness in optimization and related topics, Gargnano, (1999), Set-Valued Anal., 9 (2001), 3-11. 1, 3

[2] A. S. Antipin, N. Mijajlovic, M. Jacimovic, A second-order iterative method for solving quasi-variational inequalities, (Russian); translated from Zh. Vychisl. Mat. Mat. Fiz., 53 (2013), 336-342, Comput. Math. Math. Phys., 53 (2013), 258-264.

[3] A. S. Antipin, N. Miyaullovich, M. Yachimovich, A second-order continuous method for solving quasi-variational inequalities, (Russian); translated from Zh. Vychisl. Mat. Mat. Fiz., 51 (2011), 1973-1980, Comput. Math. Math. Phys., 51 (2011), 1856-1863.

[4] C. Baiocchi, A. C. Capelo, Variational and quasivariational inequalities: Applications to free boundary problems, John Wiley \& Sons, Inc., New York, (1984). 1, 2

[5] B. Bin-Mohsin, B, M. A. Noor, K. I. Noor, R. Latif, Strongly mixed variational inequalities and dynamical systems, Preprint, (2017). 4, 4

[6] C.-H. Chen, S.-Q. Ma, J.-F. Yang, A general inertial proximal point algorithm for mixed variational inequality problem, SIAM J. Optim., 25 (2015), 2120-2142. 1, 2

[7] J. Dong, D. Zhang, A. Nagurney, A projected dynamical systems model of general financial equilibrium with stability analysis, Math. Comput. Modelling, 24 (1996), 35-44. 1

[8] P. Dupuis, A. Nagurney, Dynamical systems and variational inequalities, Advances in equilibrium modeling, analysis and computation, Ann. Oper. Res., 44 (1993), 9-42. 1

[9] T. L. Friesz, D. Bernstein, N. J. Mehta, R. L. Tobin, S. Ganjalizadeh, Day-to-day dynamic network disequilibria and idealized traveler information systems, Oper. Res., 42 (1994), 1120-1136. 1

[10] T. L. Friesz, D. Bernstein, R. Stough, Dynamic systems, variational inequalities and control theoretic models for predicting time-varying urban network flows, Transport. Sci., 30 (1996), 14-31.

[11] F. Giannessi, A. Maugeri, Variational inequalities and network equilibrium problems, Plenum Press, New York, (1995). 1,2

[12] D. Goeleven, Existence and uniqueness for a linear mixed variational inequality arising in electrical circuits with transistors, J. Optim. Theory Appl., 138 (2008), 397-406. 1

[13] D. Kinderlehrer, G. Stampacchia, An introduction to variational inequalities and their applications, Reprint of the 1980 original, Classics in Applied Mathematics, Society for Industrial and Applied Mathematics (SIAM), Philadelphia, PA, (2000). 1, 2, 2.6

[14] G. M. Korpelevich, The extragradient method for finding saddle points and other problems, Metekon, 12 (1976), 747-756. 1

[15] P. E. Maingé, M. L. Gobinddass, Convergence of one-step projected gradient methods for variational inequalities, J. Optim. Theory Appl., 171 (2016), 146-168. 1, 2

[16] N. Mijajlović, M. Jaćimović, A proximal method for solving quasi-variational inequalities, Comput. Math. Math. phys., 55 (2015), 1981-1985. 1, 2

[17] G. V. Milovanović, M. T. Rassias (Eds.), Analytic number theory, approximation theory, and special functions, In honor of Hari M. Srivastava, Springer, New York, (2014). 1

[18] A. Nagurney, D. Zhang, Projected dynamical systems and variational inequalities with applications, First edition, Kluwer Academic Publishers, New York, (1996). 1, 2

[19] J. F. Nash, Jr., M. T. Rassias (Eds.), Open problems in mathematics, Springer, [Cham], (2016). 1

[20] M. A. Noor, A Wiener-Hopf dynamical system for variational inequalities, New Zealand J. Math., 31 (2002), $173-182$. 1,2

[21] M. A. Noor, Resolvent dynamical systems for mixed variational inequalities, Korean J. Comput. Appl. Math., 9 (2002), 15-26. 2

[22] M. A. Noor, Implicit dynamical systems and quasi variational inequalities, Appl. Math. Comput., 134 (2003), 69-81. 1, 2

[23] M. A. Noor, Fundamentals of mixed quasi variational inequalities, Int. J. Pure Appl. Math., 15 (2004), 137-258. 2

[24] M. A. Noor, Some developments in general variational inequalities, Appl. Math. Comput., 152 (2004), 199-277. 1, 2

[25] M. A. Noor, M. Akhtar, K. I. Noor, Inertial proximal method for mixed quasi variational inequalities, Nonlinear Funct. Anal. Appl., 8 (2003), 489-496. 1, 3

[26] M. A. Noor, K. I. Noor, T. M. Rassias, Some aspects of variational inequalities, J. Comput. Appl. Math., 47 (1993), 285-312. 1

[27] M. Patriksson, Nonlinear programming and variational inequalities: a unified approach, Kluwer Academic Publishers, Dordrecht, Holland, (1998).

[28] I. P. Ryazantseva, First-order methods for certain quasi-variational inequalities in a Hilbert space, Comput. Math. Math. Phys., 47 (2007), 183-190.

[29] I. P. Ryazantseva, Second-order methods for some quasi-variational inequalities, (Russian); translated from Differ. Uravn., 44 (2008), 976-987, Differ. Equ., 44 (2008), 1006-1017. 
[30] G. Stampacchia, Formes bilinaires coercitives sur les ensembles convexes, (French) C. R. Acad. Sci. Paris, 258 (1964), 4413-4416. 1, 2

[31] Y.-S. Xia, J. Wang, A recurrent neural network for solving linear projection equations, Neural Netw., 13 (2000), 337-350.

[32] Y.-S. Xia, J. Wang, On the stability of globally projected dynamical systems, J. Optim. Theory Appl., 106 (2000), 129-150. 1

[33] D. Zhang, A. Nagurney, On the stability of projected dynamical systems, J. Optim. Theory Appl., 85(1995), 97-124. 1, 2 